\title{
Effects of Region, Epidemic Stage, and Demographic Characteristics on Sleep Quality and Mental Disturbances among Health Care Workers during COVID-19 Outbreak
}

\author{
Ruike Zhang \\ Second Military Medical University \\ Tianya Hou \\ Second Military Medical University \\ Xiangyu Kong \\ Huoshenshan Hospital \\ Guibin Wang \\ the Second Affiliated Hospital of Hunan Normal University \\ Hao Wang
}

Second Military Medical University

Shuyu Xu

Second Military Medical University

Jingzhou Xu

Second Military Medical University

Jingwen $\mathrm{He}$

Second Military Medical University

Lei Xiao

Second Military Medical University

Yajing Wang

Second Military Medical University

Jing Du

Second Military Medical University

Yujia Huang

Second Military Medical University

Tong Su ( $\nabla$ sutong-2006@163.com )

Second Military Medical University https://orcid.org/0000-0002-3853-8506

Yunxiang Tang

Second Military Medical University

Research article 
Keywords: COVID-19, Depression, Anxiety, PTSD, Sleep quality, Health care workers

Posted Date: May 4th, 2020

DOI: https://doi.org/10.21203/rs.3.rs-23260/v1

License: (a) (i) This work is licensed under a Creative Commons Attribution 4.0 International License. Read Full License 


\section{Abstract}

Objective To investigate the prevalence of sleep quality and mental disturbances of medical staff and identify the effects of region, epidemic stage, and demographic characteristics during the COVID-19 outbreak in China.

Method Self-administered questionnaire were sent to health care workers (HCWs) in China from 30 Jan to 2 March, 2020. The Pittsburgh Sleep Quality Index, the Patient Health Questionare-9, the Generalized Anxiety Disorder-7 and the Impact of Event Scale were used to assess sleep quality, depression symptoms, anxiety symptoms and Post-traumatic stress disorder (PTSD) of HCWs, respectively. The influencing factors of psychological and sleep disturbances were identified by univariate analysis and multiple regression. Result The incidence of people getting depression, anxiety or PTSD symptoms were $11.6 \%, 13.3 \%, 14.3 \%$, respectively. HCWs in Hubei province experienced significantly poorer sleep quality ( $t=5.034, P<0.001)$. The predictors of sleep quality among HCWs were COVID-19 stage $2(\beta=.135, p=.014)$ and $3(\beta=.184, p=.001)$, female $(\beta=.141$, $p=.003)$ and older age $(\beta=.160, p=.001)$. Not working in Hubei province $(\beta=-.264, P<0.001)$ showed to be a protective factor of sleep quality. The risk factors of depression symptoms were not working in Hubei province $(\mathrm{OR}=4.318, \mathrm{P}<0.001)$, administrative and logistic staff and others $(\mathrm{OR}=3.538, \mathrm{p}=.011)$, and higher PSQI score $(\mathrm{OR}=1.282, \mathrm{P}<0.001)$. Having children (under-age: $\mathrm{OR}=.292, \mathrm{p}=.001$, grown-up: $\mathrm{OR}=.293, \mathrm{p}=.042)$ was identified as a protective factor of having depression symptoms. Poor sleep quality showed to be the risk factor of anxiety and PTSD symptoms as well (both $\mathrm{p}<.001$ ). Furthermore, administrative and logistic staff and others (OR=3.399, $\mathrm{p}=.006$ ) were found to be the risk factor of PTSD symptoms among HCWs.

Conclusion HCWs had poorer sleep quality on stage 2and 3 of the outbreak. HCWs in Hubei had poorer sleep quality but lighter depression condition. gender, age, occupation and status of having children were associated with sleep and mental health. Mental health programs should be considered for HCWs especially those with specific characteristics. Key words COVID-19, Depression, Anxiety, PTSD, Sleep quality, Health care workers

\section{Introduction}

Several pneumonia cases caused by unknown etiology were firstly detected in Wuhan, Hubei Province of China at the end of 2019 and the World Health Organization (WHO) China Country Office was timely informed and responded to the outbreak of Novel Coronavirus (COVID-19) together with Regional Office for the Western Pacific and headquarters on January 5, 2020(1). The outbreak of COVID-19 was declared from being a Public Health Emergency of International Concern (PHEIC) to a global pandemic on 11 March by WHO Director General after the epidemic has widely spread to the rest of the world(2).Fever, cough, fatigue, diarrhea and vomiting are the major symptoms of COVID-19. At the same time, the sign of having difficulty in breathing has been found in a few cases. The infiltration in both lungs was shown on chest radiographs of the infected (3-5).

Although COVID-19 is similar to SARS-CoV and MERS-CoV as an acute respiratory disease, it is different(5). Early cases suggest that the COVID-19 may not be as severe as SARS-CoV and MERS-CoV, but it is more infectious according to rapidly increasing incidence and evidence of human-to-human transmission (6).

Health care workers (HCWs) are those at risks confronting outbreaks and pathogens unknown to date (7). There are multiple ways causing health care workers increasing risks of attracting infectious diseases (8). 
Previous Research found that workers in the medical services had a higher rate of being infected by patient contacts and had a higher prevalence of work-related morbidity (9). Working in health care industry can endanger health, using SARS as a serious reminder (10). Among all SARS patients, about one fifth of them are HCWs, and HCWs comprised more than $40 \%$ of patients in Canada and Singapore (11).

Early research concerning the outbreaks such as SARS and Ebola virus disease found that workers in healthcare system have faced with more severe emotional stress during the epidemic (12-14).

Extensive studies regarding SARS outbreak have demonstrated that 18 to $57 \%$ medical staff have endured serious emotional distress during and shortly after the epidemic[12,13,15-17].

Data from several studies have shown that natural disasters such as earthquakes and pandemics caused severe mental disorders, among which attracted most attention are posttraumatic stress, major depression and sleep disturbances (18-20). During the epidemic, medical staff usually have a high level of anxiety because of the fear of transmitting infectious diseases to family, friends and colleagues [21].

PTSD has enormous effects on nursing care providers by changing their attitudes toward professionalism which may further result in increased physical and mental distress, decreased job satisfaction, increased turnover rate and reduced efficiency. Furthermore, it would also negatively affect the nursing care quality (2225). Depression anxiety and sleep problems could also cause adverse consequences to HCWs and the work settings. Therefore, it is necessary to investigate the risk factors of mental and sleep health among HCWs during COVID-19 and specific solutions should be made targeting those who are vulnerable to the outbreak.

As the epidemic progresses to different stages, the sleep and psychological conditions of HCWs may change. No previous study has investigated the impact of the stage of epidemic on sleep quality and mental health of HCWs during the COVID-19 outbreak.

In this study, we assessed the mental health and sleep quality of HCWs. We sought to analyse the predictive effect of region, epidemic stage and sociodemographic variables on sleep quality, and together with sleep quality on anxiety, depression and PTSD symptoms during the epidemic.

As the COVID-19 continues and will continue to spread over the next few months, this research may help find those working in health care industry who are more prone to suffer from emotional and sleep problems, Which may provide a basis for further intervention.

\section{Method}

\section{Study design and Participants}

The study was a quantitative survey for HCWs in China including doctors, nurses, medical technicians, administrative, logistic staff and others working in hospital. The time span of the study was between 30 January and 2 March, 2020. All health care workers voluntarily participated, completed a questionnaire anonymously online. The study was approved by the Research Ethics Commission of Naval Medical University,. 


\section{Measures}

Sociodemographic variables were collected, such as gender, age, years of working, education level, marital status, occupation, if one is the only child in his family and child status. COVID-19 time were normalized into three stages: stage1 (30 Jan to 17 Feb), stage2 (18 Feb to 24 Feb) and stage3 (25 Feb to 2 March). The reason

why the stages have been defined is that on $18 \mathrm{Feb}$, for the first time, the number of new cases was less than the number of new cured cases. The number of confirmed cases rose to the first peak in China between 24 Feb to $28 \mathrm{Feb}(26)$. So we chose $25 \mathrm{Feb}$ as the second cut-off point.

The Pittsburgh Sleep Quality Index (PSQI) was used in this study to test HCWs' sleep quality. It is a selfadministered scale including 19-items which consists of 7 dimensions including subjective sleep quality, sleep latency, sleep duration, sleep efficiency, sleep disturbances, use of sleeping medications and daytime dysfunction. The PSQI global score ranges from 0 to 21 with higher score indicating worse sleep quality(27).

The Patient Health Questionare-9 (PHQ-9) was used to detect depression among HCWs in this study. Each item of the scale were scored from 0 to 3 , with total PHQ-9 score ranging from 0 to 27 . A cut-off point of 10 was used in this study with high specificity (88\%) and sensitivity(88\%). Scores $\geq 10$ are recognized as the presence of major depression symptoms(28).

The Generalized Anxiety Disorder-7 (GAD-7) was administered to participants to test anxiety scores. The total GAD-7 score ranges from 0 to 21 . Those whose score $\geq 8$ were diagnosed to have anxiety symptoms. The sensitivity (77\%) and specificity (82\%) are appropriate(29).

The Impact of Event Scale (IES-R) scale was used to assess subjective stress caused by traumatic event. It includes 22 items and consists of 3 subscales which are intrusive, avoidance and hyperarousal. The scale ranges from 0 to 88 . IES-R total score $₫ 33$ are identified to have psychological stress(30).

The selected 3 questionnaires have Cronbach's a coefficients of more than 0.80 which shows good internal consistency $(28-30)$.

\section{Statistical analysis}

Depression, anxiety, and PTSD scales were converted to dichotomous variables because the scale values were not normally distributed.

The group comparisons of categorical variables were conducted by Chi-square test and continuous variables by T-test.

Simple linear regression was used for univariate analysis of PSQI total score. For univariate analysis of depression syndrome, anxiety syndrome and PTSD syndrome, Chi-square test and Fisher's Exact test were used for categorical variables and simple logistic regression were used for interval variables.

For categorical variables, the count and frequency were presented and the internal variables were summarized as mean \pm standard deviation. 
Thirdly, variables with $p \llbracket 0.2$ in univariate analysis were subjected to multiple regression analysis. multiple linear regression with stepwise method was performed for predicting risk factors of PSQI score. Multiple logistic regression analysis with forward: conditional procedure was conducted for detecting risk factors of Depression syndrome, Anxiety syndrome and PTSD syndrome.

Two-sided $P$ value $<0.05$ was identified as statistically significant. All statistical analyses were performed using SPSS 26.0 (Statistical Package for the Social Sciences) for Windows (SPSS, Chicago, IL).

\section{Result}

\section{Baseline information}

421 respondents completed the questionnaire. There are 59 (14\%) participants existing missing values over sleep efficiency. Expectation Maximization (EM) interpolation method was used to fill in the missing values. Among all the HCW's (421) participating in the study, 177 (42\%) of them were working in Hubei province, which is the epicenter of the ongoing outbreak in China. Most of the respondents in our study were female (291, $69.1 \%)$, and nurses $(203,48.2 \%)$. We divided the time span of the study into 3 stages, and we have 163 (38.7\%) respondents in stage 1, $133(31.6 \%)$ respondents in stage 2 and $125(29.7 \%)$ in stage 3 . The mean age of the participants were $35.48(S D=8.543)$ years old. The average years of working of participants were 14.05 $(S D=9.687)$ years. The mean PSQI score of the subjects were $5.82(S D=3.315)$. The data showed that the number of people with depression, anxiety or PTSD symptoms was 49 (11.6\%), 56 (13.3\%), 60 (14.3\%), respectively(Table 1). 
Table 1

Sociodemographic variables of HCWs

\begin{tabular}{|c|c|c|}
\hline Characteristics & N or Mean & Frequency or SD \\
\hline \multicolumn{3}{|l|}{ Hubei Province } \\
\hline Hubei & 177 & $42.0 \%$ \\
\hline Non-Hubei & 244 & $58.0 \%$ \\
\hline \multicolumn{3}{|l|}{ COVID-19 stage } \\
\hline Stage 1 & 163 & $38.7 \%$ \\
\hline Stage 2 & 133 & $31.6 \%$ \\
\hline Stage 3 & 125 & $29.7 \%$ \\
\hline \multicolumn{3}{|l|}{ Gender } \\
\hline Male & 130 & $30.9 \%$ \\
\hline Female & 291 & $69.1 \%$ \\
\hline \multicolumn{3}{|l|}{ Occupation } \\
\hline Doctor & 118 & $28.0 \%$ \\
\hline Nurse & 203 & $48.2 \%$ \\
\hline Medical Technician & 47 & $11.2 \%$ \\
\hline Administrative and logistic staff and others & 53 & $12.6 \%$ \\
\hline \multicolumn{3}{|l|}{ Education } \\
\hline Associate & 70 & $16.6 \%$ \\
\hline Bachelor's & 279 & $66.3 \%$ \\
\hline Master's/Doctorate & 72 & $17.1 \%$ \\
\hline \multicolumn{3}{|l|}{ Only child in one's family } \\
\hline Yes & 143 & $34.0 \%$ \\
\hline No & 278 & $66.0 \%$ \\
\hline \multicolumn{3}{|l|}{ Marital status } \\
\hline Married & 289 & $68.6 \%$ \\
\hline Other & 132 & $31.4 \%$ \\
\hline \multicolumn{3}{|l|}{ Child Status } \\
\hline No Child & 148 & $35.2 \%$ \\
\hline Under aged children & 236 & $56.1 \%$ \\
\hline
\end{tabular}




\begin{tabular}{|lll|}
\hline Characteristics & N or Mean & Frequency or SD \\
\hline Grown-up children & 37 & $8.8 \%$ \\
\hline Age & 35.48 & 8.543 \\
\hline Years of working & 14.05 & 9.687 \\
\hline PSQI (Sleep quality) & 5.82 & 3.315 \\
\hline Depression & & \\
Yes & 49 & $11.6 \%$ \\
\hline No & 372 & $88.4 \%$ \\
\hline Anxiety & & \\
\hline Yes & 56 & $13.3 \%$ \\
\hline No & 365 & $86.7 \%$ \\
\hline PTSD & & \\
Yes & 60 & $14.3 \%$ \\
\hline No & 361 & $85.7 \%$ \\
\hline
\end{tabular}

\section{Differences Between Hubei And Non-hubei Group}

We tested the interregional differences of PSQI, depression, anxiety, PTSD and component scores between Hubei and Non-Hubei groups. The difference of PSQI global score between Hubei $(6.77 \pm 3.482)$ and NonHubei $(5.13 \pm 3.013)$ group is highly significant $(P<.001)$. The scores in Hubei group was significantly higher in Subjective sleep quality $(t=3.267, p=.001)$, Sleep duration $(t=6.780, p<.001)$, Habitual sleep efficiency $(t=$ $2.919, p=.004)$, Use of sleeping medications $(t=2.302, p=.022)$ and Daytime dysfunction $(t=3.369, p=.001)$. The difference of depression syndrome between Hubei and Non-Hubei groups are significant $(p=.001)$. The differences of anxiety syndrome $(p=.303)$ or psychological stress $(p=.362)$ were not significant between groups (supplementary Table 1).

\section{Sleep Quality And Mental Health Across Different Covid- 19epidemic Stages}

The sleep quality of HCWs was significantly worse in COVID-19 epidemic stage $2(\beta=.135, p=.014)$ and stage $3(\beta=.184, p=.001)$. While depression, anxiety and PTSD symptoms had no significant differences over time (all p囚.05) (Fig. 1 and Tables 2 and 3). 
Table 2

Univariate and multivariate analysis of influencing factors of sleep quality

\begin{tabular}{|c|c|c|c|c|c|c|}
\hline \multirow[t]{2}{*}{ Variables } & \multicolumn{3}{|c|}{ Univariate } & \multicolumn{3}{|c|}{ Multivariate } \\
\hline & $\beta$ & $\mathrm{B}(\mathrm{Cl})$ & $\mathrm{p}$ & $\beta$ & $\mathrm{B}(\mathrm{Cl})$ & $\mathrm{p}$ \\
\hline Age & .097 & $\begin{array}{l}.038(.000 \\
.075)\end{array}$ & .048 & .160 & $\begin{array}{l}.062(.025 \\
.099)\end{array}$ & .001 \\
\hline \multicolumn{3}{|l|}{ Hubei Province } & \multicolumn{4}{|l|}{.000} \\
\hline Hubei & \multicolumn{3}{|l|}{ ref } & \multicolumn{3}{|l|}{ ref } \\
\hline Non-Hubei & -.244 & $\begin{array}{l}-1.637(-2.261 \\
-1.012)\end{array}$ & & -.264 & $\begin{array}{l}-1.773 \\
(-2.390, \\
-1.156)\end{array}$ & .000 \\
\hline \multicolumn{3}{|l|}{ COVID-19 stage } & \multicolumn{4}{|l|}{.046} \\
\hline Stage 1 & \multicolumn{3}{|l|}{ ref } & \multicolumn{3}{|l|}{ ref } \\
\hline Stage 2 & .065 & $\begin{array}{l}.465(-.292 \\
1.223)\end{array}$ & .228 & .135 & $\begin{array}{l}.961(.196, \\
1.726)\end{array}$ & .014 \\
\hline Stage 3 & .136 & $\begin{array}{l}.988(.218 \\
1.759)\end{array}$ & .012 & .184 & $\begin{array}{l}1.336(.586, \\
2.085)\end{array}$ & .001 \\
\hline \multicolumn{3}{|l|}{ Gender } & \multicolumn{4}{|l|}{.005} \\
\hline Male & \multicolumn{3}{|l|}{ ref } & \multicolumn{3}{|l|}{ ref } \\
\hline Female & .135 & $\begin{array}{l}.968(.287, \\
1.650)\end{array}$ & & .141 & $\begin{array}{l}1.011(.349 \\
1.674)\end{array}$ & .003 \\
\hline \multicolumn{3}{|l|}{ Occupation } & \multicolumn{4}{|l|}{.014} \\
\hline Doctor & \multicolumn{6}{|l|}{ ref } \\
\hline Nurse & .171 & $\begin{array}{l}1.130(.383, \\
1.878)\end{array}$ & \multicolumn{4}{|l|}{.003} \\
\hline Medical Technician & .017 & $\begin{array}{l}.183(-.930 \\
1.297)\end{array}$ & \multicolumn{4}{|l|}{.747} \\
\hline $\begin{array}{l}\text { Administrative and logistic staff } \\
\text { and others }\end{array}$ & .022 & $\begin{array}{l}.217(-.850, \\
1.285)\end{array}$ & \multicolumn{4}{|l|}{.689} \\
\hline Education & & & \multicolumn{4}{|l|}{.621} \\
\hline \multicolumn{7}{|c|}{$\begin{array}{l}\text { Predictive variables tested by multiple linear regression (stepwise method): Hubei Province, COVID-19 } \\
\text { stage, Gender, Occupation, Marital status, Child Status, Age and years of working }\end{array}$} \\
\hline \multicolumn{7}{|l|}{ Outcome: PSQI total score } \\
\hline \multicolumn{7}{|c|}{ Constant $=4.004$, Adjusted $R^{2}=0.109, F=11.206, p=0.000$} \\
\hline \multicolumn{7}{|l|}{ Ref: Reference } \\
\hline Bold p: significant & & & & & & \\
\hline
\end{tabular}




\begin{tabular}{|c|c|c|c|}
\hline Variables & \multicolumn{2}{|c|}{ Univariate } & Multivariate \\
\hline Associate & \multicolumn{3}{|l|}{ ref } \\
\hline Bachelor's & -.034 & $\begin{array}{l}-.237(-1.110, \\
.635)\end{array}$ & .593 \\
\hline Master's/Doctorate & -.062 & $\begin{array}{l}-.541(-1.636 \\
.554)\end{array}$ & .332 \\
\hline \multicolumn{3}{|l|}{ Only child in one's family } & .977 \\
\hline Yes & \multicolumn{3}{|l|}{ ref } \\
\hline No & -.001 & $\begin{array}{l}-.010(-.681 \\
.661)\end{array}$ & .977 \\
\hline \multicolumn{3}{|l|}{ Marital status } & .101 \\
\hline Married & \multicolumn{3}{|l|}{ ref } \\
\hline Other & .080 & $\begin{array}{l}.532(-.105 \\
1.168)\end{array}$ & .101 \\
\hline \multicolumn{3}{|l|}{ Child Status } & .125 \\
\hline No Child & \multicolumn{3}{|l|}{ ref } \\
\hline Under aged children & .086 & $\begin{array}{l}.577(-.105 \\
1.258)\end{array}$ & .097 \\
\hline Grown-up children & .088 & $\begin{array}{l}1.032(-.162 \\
2.227)\end{array}$ & .090 \\
\hline Years of working & .101 & $.034(.002$ & .039 \\
\hline \multicolumn{4}{|c|}{$\begin{array}{l}\text { Predictive variables tested by multiple linear regression (stepwise method): Hubei Province, COVID-19 } \\
\text { stage, Gender, Occupation, Marital status, Child Status, Age and years of working }\end{array}$} \\
\hline \multicolumn{4}{|l|}{ Outcome: PSQI total score } \\
\hline \multicolumn{4}{|c|}{ Constant $=4.004$, Adjusted $R^{2}=0.109, F=11.206, p=0.000$} \\
\hline \multicolumn{4}{|l|}{ Ref: Reference } \\
\hline \multicolumn{4}{|l|}{ Bold p: significant } \\
\hline
\end{tabular}


Table 3

Univariate analysis of influencing factors of depression syndrome, anxiety syndrome and PTSD syndrome

\begin{tabular}{|c|c|c|c|c|c|c|c|c|c|}
\hline \multirow[b]{2}{*}{ Characteristics } & \multicolumn{3}{|c|}{ Depression } & \multicolumn{3}{|c|}{ Anxiety } & \multicolumn{3}{|l|}{ PTSD } \\
\hline & Yes & No & $\mathrm{p}$ & Yes & No & $\mathrm{p}$ & Yes & No & $\mathrm{p}$ \\
\hline \multicolumn{10}{|l|}{$\begin{array}{l}\text { Categorical } \\
\text { variable }\end{array}$} \\
\hline Hubei Province & & & .001 & & & .303 & & & .362 \\
\hline Hubei & $\begin{array}{l}10 \\
(20.4)\end{array}$ & $\begin{array}{l}167 \\
(44.9)\end{array}$ & & $\begin{array}{l}20 \\
(35.7)\end{array}$ & $\begin{array}{l}157 \\
(43.0)\end{array}$ & & $\begin{array}{l}22 \\
(36.7)\end{array}$ & $\begin{array}{l}155 \\
(42.9)\end{array}$ & \\
\hline Non-Hubei & $\begin{array}{l}39 \\
(79.6)\end{array}$ & $\begin{array}{l}205 \\
(55.1)\end{array}$ & & $\begin{array}{l}36 \\
(64.3)\end{array}$ & $\begin{array}{l}208 \\
(57.0)\end{array}$ & & $\begin{array}{l}38 \\
(63.3)\end{array}$ & $\begin{array}{l}206 \\
(57.1)\end{array}$ & \\
\hline COVID-19 period & & & .062 & & & .411 & & & .690 \\
\hline Stage 1 & $\begin{array}{l}18 \\
(36.7)\end{array}$ & $\begin{array}{l}145 \\
(39.0)\end{array}$ & & $\begin{array}{l}19 \\
(33.9)\end{array}$ & $\begin{array}{l}144 \\
(39.5)\end{array}$ & & $\begin{array}{l}25 \\
(41.7)\end{array}$ & $\begin{array}{l}138 \\
(38.2)\end{array}$ & \\
\hline Stage 2 & $\begin{array}{l}22 \\
(44.9)\end{array}$ & $\begin{array}{l}111 \\
(29.8)\end{array}$ & & $\begin{array}{l}22 \\
(39.3)\end{array}$ & $\begin{array}{l}111 \\
(30.4)\end{array}$ & & $\begin{array}{l}20 \\
(33.3)\end{array}$ & $\begin{array}{l}113 \\
(31.3)\end{array}$ & \\
\hline Stage 3 & $\begin{array}{l}9 \\
(18.4)\end{array}$ & $\begin{array}{l}116 \\
(31.2)\end{array}$ & & $\begin{array}{l}15 \\
(26.8)\end{array}$ & $\begin{array}{l}110 \\
(30.1)\end{array}$ & & $\begin{array}{l}15 \\
(25.0)\end{array}$ & $\begin{array}{l}110 \\
(30.5)\end{array}$ & \\
\hline Gender & & & .966 & & & .928 & & & .287 \\
\hline Male & $\begin{array}{l}15 \\
(30.6)\end{array}$ & $\begin{array}{l}115 \\
(30.9)\end{array}$ & & $\begin{array}{l}17 \\
(30.4)\end{array}$ & $\begin{array}{l}113 \\
(31.0)\end{array}$ & & $\begin{array}{l}15 \\
(25.0)\end{array}$ & $\begin{array}{l}115 \\
(31.9)\end{array}$ & \\
\hline Female & $\begin{array}{l}34 \\
(69.4)\end{array}$ & $\begin{array}{l}257 \\
(69.1)\end{array}$ & & $\begin{array}{l}39 \\
(69.6)\end{array}$ & $\begin{array}{l}252 \\
(69.0)\end{array}$ & & $\begin{array}{l}45 \\
(75.0)\end{array}$ & $\begin{array}{l}246 \\
(68.1)\end{array}$ & \\
\hline Occupation & & & .004 & & & .163 & & & .008 \\
\hline Doctor & $\begin{array}{l}9 \\
(18.4)\end{array}$ & $\begin{array}{l}109 \\
(29.3)\end{array}$ & & $\begin{array}{l}10 \\
(17.9)\end{array}$ & $\begin{array}{l}108 \\
(29.6)\end{array}$ & & $\begin{array}{l}13 \\
(21.7)\end{array}$ & $\begin{array}{l}105 \\
(29.1)\end{array}$ & \\
\hline Nurse & $\begin{array}{l}21 \\
(42.9)\end{array}$ & $\begin{array}{l}182 \\
(48.9)\end{array}$ & & $\begin{array}{l}29 \\
(51.8)\end{array}$ & $\begin{array}{l}174 \\
(47.7)\end{array}$ & & $\begin{array}{l}29 \\
(48.3)\end{array}$ & $\begin{array}{l}174 \\
(48.2)\end{array}$ & \\
\hline $\begin{array}{l}\text { Medical } \\
\text { Technician }\end{array}$ & $\begin{array}{l}5 \\
(10.2)\end{array}$ & $\begin{array}{l}42 \\
(11.3)\end{array}$ & & $\begin{array}{l}6 \\
(10.7)\end{array}$ & $\begin{array}{l}41 \\
(11.2)\end{array}$ & & $\begin{array}{l}3 \\
(5.0)\end{array}$ & $\begin{array}{l}44 \\
(12.2)\end{array}$ & \\
\hline $\begin{array}{l}\text { Administrative and } \\
\text { logistic staff and } \\
\text { others }\end{array}$ & $\begin{array}{l}14 \\
(28.6)\end{array}$ & $\begin{array}{l}39 \\
(10.5)\end{array}$ & & $\begin{array}{l}11 \\
(19.6)\end{array}$ & $\begin{array}{l}42 \\
(11.5)\end{array}$ & & $\begin{array}{l}15 \\
(25.0)\end{array}$ & $\begin{array}{l}38 \\
(10.5)\end{array}$ & \\
\hline Education & & & .027 & & & .395 & & & .435 \\
\hline Associate & $\begin{array}{l}13 \\
(26.5)\end{array}$ & $\begin{array}{l}57 \\
(15.3)\end{array}$ & & $\begin{array}{l}10 \\
(17.9)\end{array}$ & $\begin{array}{l}60 \\
(16.4)\end{array}$ & & $\begin{array}{l}13 \\
(21.7)\end{array}$ & $\begin{array}{l}57 \\
(15.8)\end{array}$ & \\
\hline Bachelor's & $\begin{array}{l}33 \\
(67.3)\end{array}$ & $\begin{array}{l}246 \\
(66.1)\end{array}$ & & $\begin{array}{l}40 \\
(71.4)\end{array}$ & $\begin{array}{l}239 \\
(65.5)\end{array}$ & & $\begin{array}{l}39 \\
(65.0)\end{array}$ & $\begin{array}{l}240 \\
(66.5)\end{array}$ & \\
\hline Bold p: significant & & & & & & & & & \\
\hline
\end{tabular}




\begin{tabular}{|c|c|c|c|c|c|c|c|c|c|}
\hline \multirow[b]{2}{*}{ Master's/Doctorate } & \multicolumn{3}{|c|}{ Depression } & \multicolumn{3}{|c|}{ Anxiety } & \multicolumn{3}{|l|}{ PTSD } \\
\hline & \multirow[t]{2}{*}{$\begin{array}{l}3 \\
(6.1)\end{array}$} & \multicolumn{2}{|l|}{$\begin{array}{l}69 \\
(18.5)\end{array}$} & \multirow[t]{2}{*}{$\begin{array}{l}6 \\
(10.7)\end{array}$} & \multicolumn{2}{|l|}{$\begin{array}{l}66 \\
(18.1)\end{array}$} & \multirow[t]{2}{*}{$\begin{array}{l}8 \\
(13.3)\end{array}$} & \multicolumn{2}{|l|}{$\begin{array}{l}64 \\
(17.7)\end{array}$} \\
\hline $\begin{array}{l}\text { Only child in one's } \\
\text { family }\end{array}$ & & & .086 & & & .070 & & & .855 \\
\hline Yes & $\begin{array}{l}22 \\
(44.9)\end{array}$ & $\begin{array}{l}121 \\
(32.5)\end{array}$ & & $\begin{array}{l}25 \\
(44.6)\end{array}$ & $\begin{array}{l}118 \\
(32.3)\end{array}$ & & $\begin{array}{l}21 \\
(35.0)\end{array}$ & $\begin{array}{l}122 \\
(33.8)\end{array}$ & \\
\hline No & $\begin{array}{l}27 \\
(55.1)\end{array}$ & $\begin{array}{l}251 \\
(67.5)\end{array}$ & & $\begin{array}{l}31 \\
(55.4)\end{array}$ & $\begin{array}{l}247 \\
(67.7)\end{array}$ & & $\begin{array}{l}39 \\
(65)\end{array}$ & $\begin{array}{l}239 \\
(66.2)\end{array}$ & \\
\hline Marital status & & & .005 & & & .287 & & & .148 \\
\hline Married & $\begin{array}{l}25 \\
(51)\end{array}$ & $\begin{array}{l}264 \\
(71.0)\end{array}$ & & $\begin{array}{l}35 \\
(62.5)\end{array}$ & $\begin{array}{l}254 \\
(69.6)\end{array}$ & & $\begin{array}{l}46 \\
(76.7)\end{array}$ & $\begin{array}{l}243 \\
(67.3)\end{array}$ & \\
\hline Other & $\begin{array}{l}24 \\
(49.0)\end{array}$ & $\begin{array}{l}108 \\
(29.0)\end{array}$ & & $\begin{array}{l}21 \\
(37.5)\end{array}$ & $\begin{array}{l}111 \\
(30.4)\end{array}$ & & $\begin{array}{l}14 \\
(23.3)\end{array}$ & $\begin{array}{l}118 \\
(32.7)\end{array}$ & \\
\hline Child Status & & & .001 & & & .312 & & & .014 \\
\hline No Child & $\begin{array}{l}28 \\
(57.1)\end{array}$ & $\begin{array}{l}120 \\
(32.3)\end{array}$ & & $\begin{array}{l}25 \\
(44.6)\end{array}$ & $\begin{array}{l}123 \\
(33.7)\end{array}$ & & $\begin{array}{l}16 \\
(26.7)\end{array}$ & $\begin{array}{l}132 \\
(36.6)\end{array}$ & \\
\hline Under aged & $\begin{array}{l}16 \\
(32.7)\end{array}$ & $\begin{array}{l}220 \\
(59.1)\end{array}$ & & $\begin{array}{l}27 \\
(48.2)\end{array}$ & $\begin{array}{l}209 \\
(57.3)\end{array}$ & & $\begin{array}{l}34 \\
(56.7)\end{array}$ & $\begin{array}{l}202 \\
(56.0)\end{array}$ & \\
\hline Grown-up children & $\begin{array}{l}5 \\
(10.2)\end{array}$ & $\begin{array}{l}32 \\
(8.6)\end{array}$ & & $\begin{array}{l}4 \\
(7.1)\end{array}$ & $\begin{array}{l}33 \\
(9.0)\end{array}$ & & $\begin{array}{l}10 \\
(16.7)\end{array}$ & $\begin{array}{l}27 \\
(7.5)\end{array}$ & \\
\hline $\begin{array}{l}\text { Continuous } \\
\text { variable }\end{array}$ & OR $(95$ & $\mathrm{Cl})$ & & OR $(95$ & $6 \mathrm{Cl})$ & & OR $(95$ & Cl) & \\
\hline Age & $\begin{array}{l}0.966 \\
1.004)\end{array}$ & .930 & .077 & $\begin{array}{l}0.980 \\
1.015)\end{array}$ & .947 & .260 & $\begin{array}{l}1.029 \\
1.061)\end{array}$ & 998, & .069 \\
\hline Years of working & $\begin{array}{l}0.980 \\
1.013)\end{array}$ & 948 & .226 & $\begin{array}{l}0.990 \\
1.020)\end{array}$ & .961 & .517 & $\begin{array}{l}1.031 \\
1.060)\end{array}$ & .004 & .024 \\
\hline PSQI & $\begin{array}{l}1.163( \\
1.267)\end{array}$ & .069 & .000 & $\begin{array}{l}1.235( \\
1.343)\end{array}$ & .137 & .000 & $\begin{array}{l}1.274( \\
1.385)\end{array}$ & .173 & .000 \\
\hline Bold p: significant & & & & & & & & & \\
\hline
\end{tabular}

Univariate analysis of influencing factors of sleep quality, depression, anxiety and PTSD

The PSQI score was significantly associated with Hubei province $(p<.001)$, gender $(p=.005)$, occupation ( $p$ $=.014)$, COVID-19 time $(p=.046)$, age $(p=.048)$ and years of working $(p=.039)$. Nurses had significantly higher PSQI scores than that of doctors $(p=.003$, Table 2$)$.

As shown in Table 3, the results showed that variables of being working in Hubei province, occupation, education level, marital status and child status were significantly associated with depression symptoms (all $p$ $<0.05)$. Variables of occupation $(p=.008)$, years of working and child status were significantly associated with 
PTSD symptom(all $p<0.05$ ). The PSQI score were highly significantly associated with depression, anxiety and PTSD symptoms (all p》.001).

\section{Risk Factors Associated With Psqi, Depression, Anxiety And Ptsd}

We put all variables identified in univariate analysis with p value smaller than 0.2 into multiple regression analysis.

For PSQI, the risk factors were listed as follows: COVID-19 stage $2(p=0.014)$ and stage3 $(p=0.001)$, older age $(p=0.001)$ and female $(p=0.003)$. The protective factor was revealed to be not working in Hubei province (beta $=-0.264, \mathrm{p}<.001$ ). The adjusted $\mathrm{R}^{2}$ of the model was 0.109 (Table 2 ).

As shown in Table 4, the risk factors of depression symptom were presented: not working in Hubei province $(O R=4.318, p<.001)$, administrative, logistic staff and others $(O R=3.538, p=0.011)$, and higher PSQI score $(O R=1.282, p<.001)$. Having under-age children $(O R=0.292, p=0.001)$ or grown up children $(O R=0.293, p=$ 0.042) were shown to be the protective factor of depression symptoms. The risk factor for anxiety were revealed to be having higher PSQI score $(O R=1.235, p<.001)$. The two risk factors of PTSD symptoms were administrative and logistic staff and others $(O R=3.399, p=.006)$ and higher PSQI score $(O R=1.295, p<.001)$. 
Table 4

Multiple logistic regression to predict depression syndrome, anxiety syndrome and PTSD syndrome

\begin{tabular}{|c|c|c|c|}
\hline Variable & OR & $(95 \% \mathrm{Cl})$ & $\mathrm{p}$ \\
\hline \multicolumn{4}{|l|}{ Depression ${ }^{1}$} \\
\hline \multicolumn{4}{|l|}{ Hubei Province } \\
\hline Hubei & 1.0 & ref & \\
\hline Non-Hubei & 4.318 & $(1.911,9.759)$ & .000 \\
\hline \multicolumn{4}{|l|}{ Occupation } \\
\hline Doctor & 1.0 & ref & \\
\hline Nurse & 1.203 & $(.496,2.915)$ & .683 \\
\hline Medical Technician & 1.428 & $(.417,4.883)$ & .570 \\
\hline Administrative and logistic staff and others & 3.538 & $(1.332,9.397)$ & .011 \\
\hline \multicolumn{4}{|l|}{ Child Status } \\
\hline No Child & 1.0 & ref & \\
\hline Under aged & .292 & $(.143, .600)$ & .001 \\
\hline Grown-up children & .293 & $(.090, .956)$ & .042 \\
\hline PSQI total score & 1.282 & $(1.155,1.423)$ & .000 \\
\hline \multicolumn{4}{|l|}{ Anxiety ${ }^{2}$} \\
\hline PSQI total score & 1.235 & $(1.137,1.343)$ & .000 \\
\hline \multicolumn{4}{|l|}{ PTSD $^{3}$} \\
\hline \multicolumn{4}{|l|}{ Occupation } \\
\hline Doctor & 1.0 & ref & \\
\hline Nurse & .980 & $(.466,2.058)$ & .957 \\
\hline Medical Technician & .467 & $(.119,1.833)$ & .275 \\
\hline Administrative and logistic staff and others & 3.399 & $(1.413,8.180)$ & .006 \\
\hline PSQI total score & 1.295 & $(1.187,1.413)$ & .000 \\
\hline
\end{tabular}

1: Dependent variable: depression syndrome

Predictive variables tested by Forward conditional method: Hubei Province, COVID-19 stage,

Occupation, Education, Only child in one's family, Marital status, Child Status, Age and PSQI total score

Constant $=-4.314$, Nagelkerke $\mathrm{R}^{2}=0.234$ 


\begin{tabular}{|l|}
\hline Variable \\
\hline 2: Dependent variable:anxiety syndrome \\
\hline $\begin{array}{l}\text { Predictive variables tested by Forward conditional method: Occupation, Only child in one's family, and PSQI } \\
\text { total score }\end{array}$ \\
\hline Constant=-3.284, Nagelkerke $\mathrm{R}^{2}=0.109$ \\
\hline 3: Dependent variable:PTSD syndrome \\
\hline $\begin{array}{l}\text { Predictive variables tested by Forward conditional method: Occupation, Marital status, Child Status, Age, } \\
\text { Years of working and PSQI total score }\end{array}$ \\
\hline Constant=-3.685, Nagelkerke R2 = 0.192 \\
\hline Bold p: significant \\
\hline
\end{tabular}

\section{Discussion}

Since the end of December, 2019, the COVID-19 has spread across the world. Hubei as the epicenter of the outbreak in China faced with the most serious situations of the pandemic. The emotional disturbances of $\mathrm{HCWs}$ were at high risk and should receive high attention. This study investigated the prevalence of sleep quality and mental disturbances of HCWs and presented the potential influencing factors including epidemic stage, region and demographic characteristics.

$49(11.6 \%), 56(13.3 \%)$ and $60(14.3 \%)$ of HCWs were shown to have depression, anxiety and PTSD symptoms in this study. The rates are lower comparing with the results of previous studies on SARS which presented that 18 to $57 \%$ of the medical staff endured serious emotional distress during and shortly after the epidemic $(12,13,15-17)$. This may be due to the experience of fighting SARS in 2003 and timely and effective psychological assistance to medical staff during this pandemic.

Few studies have explored the impact of the epidemic stage on sleep quality and mental health of HCWs. This study found that comparing withCOVID-19epidemic stage 1, the sleep quality at stage 2 and 3 were significantly poorer, which might probably be owing to the rapidly rising number of confirmed cases and severe cases over time. This result was contrary to that of Chen (2006) who found that compared with the mean score of PSQI of medical staff before caring for patients, the mean score of PSQI was significantly decreased 2 weeks and 1 month after the initiation of SARS prevention program (31). And the study of Chen also showed that the anxiety and depression level of nursing staff had also been alleviated after 2 weeks of the SARS caring program(31). However, the findings of the current study indicating that the depression and anxiety level of HCWs had no significant difference across 3 stages. The difference might be because that the epidemic stage 1 of our study was the early stage of the prevention program instead of the period before a prevention program began. And the subjects of our study including doctors, nurses, medical technicians and others, which was different with the previous study. What's more, the medical staff were facing with the different epidemics which have different epidemiological and clinical characteristics. The number of confirmed cases of COVID-19 has far outnumbered SARS outbreak and the longer asymptomatic latent period along with super spreader makes COVID-19 more tricky to handle with. And the source of infection and the patient zero have not been 
found yet, drugs and vaccines against the disease are also under development. The stage 3 can not be recognized as the stage on which the disease has been better understood or controlled.

During COVID-19 outbreak, HCWs in Hubei province had significantly poorer sleep quality $(p<.001)$. When it comes to the component scores of the PSQI, the study showed that HCWs in Hubei has worse subjective sleep quality, slightly longer sleep latency, shorter sleep duration, worse sleep efficiency, more often use of sleep medications, and daytime dysfunction. Working in Hubei province is a predictor of poor sleep quality. The finding was consistent with that of Grainne M McAlonan et al (2007) who found that High-risk HCWs were more risky of being fatigue and having poor sleep quality during the outbreak of SARS (32). Hubei Province is the center of the epidemic where confirmed cases and severe cases are significantly beyond those in other provinces. Therefore, the workload and work intensity of medical personnel in Hubei Province will be much higher than those in other provinces. Research found that higher workload of nurses were related to poorer sleep quality which could explain the result of the study (33). In addition, female and older age were two well established risk factors for sleep disturbances in our study and previous studies (34).

This study found that HCWs working in or outside Hubei province had equal level of prevalence to have psychological stress and anxiety symptoms. Previous studies also indicated that similar psychological morbidity and perceived stress were found between high-risk and low-risk HCWs (12,32).COVID-19 has a long incubation period and the virus carriers are undetectable and could transmit virus during the latent period. Although non-Hubei provinces are not as severe as Hubei province, the number of confirmed cases was rising rapidly as well. The HCWs outside Hubei provinces did not recognize themselves as exempt from the danger. Moreover, medical staff in Hubei has higher vigilance and confidence as the attention of the whole country was focused on the epidemic in Hubei. Thus medical staff in Hubei had a morale and sense of responsibility to conquer this challenge, which is beneficial for their mental health maintenance. These reasons may help explain the unusual result that HCWs not working in Hubei province had higher risk of depression.

It has not been described before that the status of having children is associated with depression of HCWs. This study showed that having under-age or grown-up children was a protective factor of depression symptom among HCWs. Previous research found that people with older age $(46.65 \pm 13.82)$ and coexisting conditions (28.8\%) were more likely to be affected by COVID-19 (35). Children were not the main affected group of this outbreak. And the postpone of the reopening of schools and lockdown of the cities made children stayed in home under supervision. These might explain part of the results but still acquire further studies.

Surprisingly, our study suggested that administrative and logistic staff and others working in health care industry had higher prevalence of being depression or PTSD compared with that of doctors. This finding is contrary to previous studies which have suggested that being a nursing professional and providing direct care with SARS patients were the risk factors of high level of job-related stress $(12,36)$. But the results of our study accords with earlier observations. Attack rate among HCWs during SARS varied by occupations. The attack rate of HCWs in Vietnam, 2003, were $16 \%, 35 \%, 2 \%$ for doctors, nurses and administrative staff, respectively. Those with highest attack rate was named as "other staff with patient contact" which accounted for $53 \%$ (37). A study in Hongkong during SARS outbreak reported the attack rate as follows: nurses (1.21\%), medical staff and technicians $(0.29 \%)$, and others including assistants and cleaners $(2.72 \%)(38)$. The studies above have indicated that the logistic and administrative staff and others was the most vulnerable groups that withstand 
the highest attack of SARS virus. This might be one of the reasons why administrative and logistic staff in our study endured with higher prevalence of depression and PTSD. Another reason could be that the logistic staff and others working in hospital might not be as psychologically prepared as doctors and nurses. Furthermore, situations of low exposure can also be risky of getting affected (37) as there were asymptomatic carriers. Administrative and logistic staff and others like cleaners are staff that are easily neglected in a outbreak. We should be more concerned about their mental health.

Our data showed that the risk of having depression, anxiety or PTSD symptoms tended to increase with enhancing PSQI score among HCWs. Previous study found that Service members and veterans who had higher PTSD, depression and anxiety scores were those who had relatively bad or very bad sleep quality (39). A study among South Korean workers found that the variance in weekday and weekend sleep duration are highly associated with PHQ-9 score which indicated depression (40). A recent research suggested that poor sleep quality and social support lead to anxiety, stress and self-efficacy problems among HCWs in China dealing with COVID-19 during early stages of the outbreak (41), which is consistent with our study. Several studies found that insomnia was often accompanied by mental disorders, especially anxiety and depression (42, 43). In fact, longitudinal studies have found that risk of depression and anxiety increased when insomnia existed (44-47) which would even cause the relapses (48). A considerable number of longitudinal studies (49-52) and a meta-analysis (53) suggested that people with sleep disturbances, compared with those without sleep difficulties, have over twice times risk of getting depression symptoms over the next one to three years of follow-up. Insomniac patients with depression have higher prevalence to remain depressed despite standard therapy (54). In this case, sleep disturbances are likely to be the risk factor and consequence of anxiety and depression. In this case, we could deal with mental health problems by coping with sleep disturbances which is less-stigmatizing.

The study has several limitations. Firstly, The study is a cross-sectional study, which can not investigate the causal relationship. Secondly, there is self-report bias because all results were from self-reported questionnaires. And setting up a true control group is impossible for our study for all the HCWs in China who were influenced by the COVID-19 outbreak. Thirdly, the sample of the study is relatively small which might influence the generalization of the results.

\section{Conclusions}

Our study indicated the predictors of mental disturbances and sleep quality among HCWs including epidemic stage, region, and sociodemographic variables. We hope the results could be helpful for psychological professionals and policy makers to develop specific policies and mental health advice for HCWs especially those with specific characteristics.

\section{Abbreviations}

HCWs

Health care workers

PTSD

Post-Traumatic Stress Disorder 
PSQI

The Pittsburgh Sleep Quality Index

PHQ-9

the Patient Health Questionare-9

IES-R

the Impact of Event Scale

GAD-7

the Generalized Anxiety Disorder-7

PHEIC

Public Health Emergency of International Concern

WHO

World Health Organization

\section{Declarations}

\section{Acknowledgements}

The authors would like to thank all participants, and cooperative authors in this study.

Authors' contributions

RZ participated in conception, design of the work, data interpretation and analysis, drafting and revision of the manuscript. TH, XK, JH and LX participated in the acquisition and interpretation of data. GW, HW, SX, JD, YW, $\mathrm{YH}$ and $\mathrm{JX}$ participated in the data analysis and revision of the draft. YT and TS made contribution to the concept and design of the study, acquisition of data, manuscript revision and supervision. All authors approved this final version to be published.

Funding

This research received fundings from the key scientific and research projects of logistics in PLA (AWS16J033), The General Program of National Natural Science Foundation of China (81372122), Military Medical Science and Technology Youth cultivation plan (2019QNP014) and National Natural Science Foundation of China (81602734).

Availability of data and materials

The datasets and/or analyses from the study will be available from the authors if requested reasonably.

Ethics approval and consent to participate

This study was approved by the Ethics Committee of the Naval Medical University,. The study obtained the consent of all participants.

Consent for publication

Not applicable. 
Competing interests

The authors declare that there is no competing interests in this study.

Author details

1. College of Psychology, Naval Medical University, Shanghai, China Ruike Zhang: zrk_2015@163.com, Tianya Hou: liumi9512@126.com, Hao Wang: 15021591161@163.com, Shuyu Xu: 1272442183@qq.com, Jingzhou Xu: xu_psy@163.com, Jingwen He: 784471783@qq.com, Lei Xiao: xiaolei0237@163.com, Yajing Wang: 13162309006@163.com, Jing Du: 1525091615@qq.com, Yujia Huang: 449709261@qq.com

2. Huoshenshan Hospital, Wuhan, Hubei,430100, China.xiaoniu185@qq.com.

3. Department of Neurology, The Second Affiliated Hospital of Hunan Normal University (The 921 Hospital of the Chinese PLA Joint Logistic Support Force), Changsha, Hunan, China.714010298@qq.com

* Correspondence: Yunxiang Tang: tangyun7633@sina.com, Tong Su: sutong-2006@163.com

\section{References}

1. World Health Organization.Pneumonia of unknown cause - China. https://www.who.int/csr/don/05january-2020-pneumonia-of-unkown-cause-china/en/. Updated January5, 2020.Accessed March1, 2020.

2. World Health Organization.WHO Director-General's opening remarks at the media briefing on COVID-1911. March 2020. https://www.who.int/dg/speeches/detail/who-director-general-s-opening-remarks-at-themedia-briefing-on-covid-19--11-march-2020. Updated March11, 2020.Accessed March15, 2020.

3. Chen N, Zhou M, Dong X, Qu J, Gong F, Han Y, et al. Epidemiological and clinical characteristics of 99 cases of 2019 novel coronavirus pneumonia in Wuhan, China: a descriptive study. Lancet (London, England). 2020.

4. Huang C, Wang Y, Li X, Ren L, Zhao J, Hu Y, et al. Clinical features of patients infected with 2019 novel coronavirus in Wuhan, China. London: Lancet; 2020.

5. Zhu N, Zhang D, Wang W, Li X, Yang B, Song J, et al. A Novel Coronavirus from Patients with Pneumonia in China, 2019. The New England journal of medicine. 2020.

6. WangC HornbyPW, HaydenFG GaoGF. A novel coronavirus outbreak of global health concern. Lancet. 2020. DOI:10.1016/S0140-6736(20)30185-9.

7. Berger SA. GIDEON: a comprehensive Web-based resource for geographic medicine. Int J Health Geogr. $2005 ; 22 ; 4(1): 10$.

8. Kam JK, Chan E, Lee A, Wei VW, Kwok KO, Lui D, Yuen RK. Student nurses' ethical views on responses to the severe acute respiratory syndrome outbreak. Nurs Ethics. 2020;27:969733019895804.

9. Jung SY. How to prevent transmission of infectious agents in healthcare settings. Hanyang Med Rev. 2011;31:190-9.

10. Koh D, Lim MK, Chia SE. SARS: health care work can be hazardous to health. Occup Med (Lond). 2003;53:241-3. 
11. Koh D, Sng J. Lessons from the past: perspectives on severe acute respiratory syndrome. Asia Pac J Public Health. 2010;22(3 Suppl):132S-136S.

12. Tam CW, Pang EP, Lam LC, Chiu HF. Severe acute respiratory syndrome (SARS) in Hong Kong in 2003 : stress and psychological impact among frontline healthcare workers. Psychol Med. 2004;34(7):1197204.

13. Maunder RG, Lancee WJ, Rourke S, Hunter JJ, Goldbloom D, Balderson K, et al. Factors associated with the psychological impact of severe acute respiratorysyndrome on nurses and other hospital workers in Toronto. Psychosom Med. 2004;66(6):938-42.

14. Lancee WJ, Maunder RG, Goldbloom DS. Prevalence of psychiatric disorders among Toronto hospital workers one to two years after the SARS outbreak. Psychiatr Serv. 2008;59(1):91-5.

15. Chan AO, Huak CY. Psychological impact of the 2003 severe acute respiratory syndrome outbreak on health care workers in a medium size regional general hospital in Singapore. Occup Med. 2004;54(3):190-6.

16. Phua DH, Tang HK, Tham KY. Coping responses of emergency physicians and nurses to the 2003 severe acute respiratory syndrome outbreak. Acad Emerg Med. 2005;12(4):322-8.

17. Nickell LA, Crighton EJ, Tracy CS, Al-Enazy H, Bolaji Y, Hanjrah S, et al. Psychosocial effects of SARS on hospital staff: survey of a large tertiary care institution. CMAJ. 2004;170(5):793-8.

18. Chou FH, Su TP, Chou P, et al. Survey of psychiatric disorders after an earthquake: MINI diagnosis for a village population in Taiwan. Journal Formosan Medical Association. 2005;104:305-18.

19. Goenjian AK, Steinberg AM, Najarian LM, et al. Prospective study of posttraumatic stress, anxiety, and depression reactions after earthquake and political violence. The American Journal of Psychiatry. 2000;157:911-6.

20. Mellman TA, David D, Kulick-Bell R, et al. Sleep disturbance and its relationship to psychiatric morbidity after Hurricane Andrew. The American Journal of Psychiatry. 1995;152:1659-63.

21. Maunder R, Hunter J, Vincent L, et al. The immediate psychological and occupational impact of the 2003 SARS outbreak in a teaching hospital. 2003.http://www.cmaj.ca.

22. Collins S, Long A. Too tired to care? The psychological effects of working with trauma. J Psychiatr Ment Health Nurs. 2003;10:17-27.

23. Mclvor R, Canterbury R, Gunn J. Psychological care of staff following traumatic incidents at work. The Psychiatrist. 1997;21:176-8.

24. Donnelly E, Siebert D. Occupational risk factors in the emergency medical services. Prehospital Disaster Medicine. 2009;24(5):422-9.

25. Gates D, Gillespie G, Succop P. Violence against nurses and its impact on stress and productivity. Nursing Economics\$. 2011;29(2):59-67.

26. Epidemiology of novel coronavirus pneumonia response mechanism in China CDC. Epidemiological characteristics of novel coronavirus pneumonia [J/OL]. Chinese Journal of Epidemiology. 2020,41 (202002-17).http://rs.yiigle.com/yufabiao/1181998.htm.

27. Buysse DJ, Reynolds CF, Monk TH, Berman SR, Kupfer DJ. Te Pittsburgh sleep quality index: A new instrument for psychiatric practice and research. Psychiatry Res. 1989;28:193-213. 
28. Kroenke K, Spitzer RL, Williams JB. The PHQ-9: validity of a brief depression severity measure. J Gen Intern Med. 2001;16:606-13.

29. Kroenke K, Spitzer RL, Williams JB, Monahan PO, Lowe B. Anxiety disorders in primary care: prevalence, impairment, comorbidity, and detection. Ann Intern Med. 2007;146:317-25.

30. Asif IM, Price DE, Ewing A, Rao AL, Harmon KG, Drezner JA. The impact of diagnosis: measuring the psychological response to being diagnosed with serious or potentially lethal cardiac disease in young competitive athletes. Br J Sports Med. 2016;50:163-6.

31. Chen R, Chou KR, Huang YJ, Wang TS, Liu SY, Ho LY. Effects of a SARS prevention programme in Taiwan on nursing staff's anxiety, depression and sleep quality: a longitudinal survey. Int J Nurs Stud. 2006;43(2):215-25.

32. McAlonan GM, Lee AM, Cheung V, Cheung C, Tsang KW, Sham PC, Chua SE, Wong JG. Immediate and sustained psychological impact of an emerging infectious disease outbreak on health care workers. Can J Psychiatry. 2007;52(4):241-7.

33. Gillet N, Huyghebaert T, Réveillère C, Colombat P, Fouquereau E. The Effects of Job Demands on Nurses' Burnout and Presenteeism through Sleep Quality and Relaxation. J Clin Nurs. 2019. doi:10.1111/jocn.15116.

34. Li N, Xu G, Chen G, Zheng X. Sleep quality among Chinese elderly people: A population-based study. Arch Gerontol Geriatr. 2019;87:103968.

35. Zhang X, Cai H, Hu J, Lian J, Gu J, Zhang S, Ye C, Lu Y, Jin C, Yu G, Jia H, Zhang Y, Sheng J, Li L, Yang Y. Epidemiological, clinical characteristics of cases of SARS-CoV-2 infection with abnormal imaging findings. Int J Infect Dis. 2020.

36. Maunder R. The experience of the 2003 SARS outbreak as a traumatic stress among frontline healthcare workers in Toronto: lessons learnt. Philos Trans R Soc Lond B Biol Sci. 2004;359:1117-25.

37. World Health Organization. Consensus document on the epidemiology of severe acute respiratory syndrome (SARS). http://www.who.int/csr/sars/en/WHOconsensus.pdf. Updated October 17, 2003.Accessed March 9, 2020.

38. Lau JTF, Yang X, Leung P-C, et al. SARS in three categories of hospital workers, Hong Kong. Emerg Infect Dis. $2004 ; 10: 1399-404$.

39. Plumb TR, Peachey JT, Zelman DC. Sleep disturbance is common among servicemembers and veterans of Operations Enduring Freedom and Iraqi Freedom. Psychol Serv. 2014;11(2):209-19.

40. Han KT, Kim SJ. Instability in daily life and depression: The impact of sleep variance between weekday and weekend in South Korean workers. Health Soc Care Community. 2019.

41. Xiao H, Zhang Y, Kong D, Li S, Yang N. The Effects of Social Support on Sleep Quality of Medical Staff Treating Patients with Coronavirus Disease 2019 (COVID-19) in January and February 2020 in China. Med Sci Monit. 2020;26:e923549.

42. Ohayon MM. Prevalence and comorbidity of sleep disorders in general population.Rev Prat. $2007 ; 57$ (14), $1521-1528$.

43. Harvey A, Hairston I, Gruber J, Gershon A Anxiety and sleep. Oxford Handbook of Anxiety and Related Disorders. Oxford University Press. 2008. 
44. Breslau N, Roth T, Rosenthal L, Andreski P. Sleep disturbance and psychiatric disorders: a longitudinal epidemiological study of young adults. Biol Psychiatry. 1996;39(6):411-8.

45. Ohayon MM, Roth T. Place of chronic insomnia in the course of depressive and anxiety disorders. J Psychiatr Res. 2003;37(1):9-15.

46. Roane BM, Taylor DJ. Adolescent insomnia as a risk factor for early adult depression and substance abuse. Sleep. 2008;31(10):1351-6.

47. Neckelmann D, Mykletun A, Dahl AA. Chronic insomnia as a risk factor for developing anxiety and depression. Sleep. 2007;30(7):873-80.

48. Jansson-Fröjmark M, Lindblom K. A bidirectional relationship between anxiety and depression, and insomnia? a prospective study in the general population. J Psychosom Res. 2008;64(4):443-9.

49. Chang PP, Ford DE, Mead LA, Cooper-Patrick L, Klag MJ. Insomnia in young men and subsequent depression. The Johns Hopkins Precursors Study. Am J Epidemiol. 1997;146(2):105-14.

50. Jaussent I, Bouyer J, Ancelin ML, et al. Insomnia and daytime sleepiness are risk factors for depressive symptoms in the elderly. Sleep. 2011;34(8):1103-10.

51. Fong SY, Wing YK. Longitudinal follow up of primary insomnia patients in a psychiatric clinic. Aust N Z J Psychiatry. 2007;41(7):611-7.

52. Buysse DJ, Angst J, Gamma A, Ajdacic V, Eich D, Rössler W. Prevalence, course, and comorbidity of insomnia and depression in young adults. Sleep. 2008;31(4):473-80.

53. Baglioni C, Battagliese G, Feige B, et al. Insomnia as a predictor of depression: a meta-analytic evaluation of longitudinal epidemiological studies. J Affect Disord. 2011;135(1-3):10-9.

54. Pigeon WR, Hegel M, Unutzer J, et al. Is insomnia a perpetuating factor for late-life depression in the IMPACT cohort? Sleep. 2008;31(4):481-8.

\section{Figures}




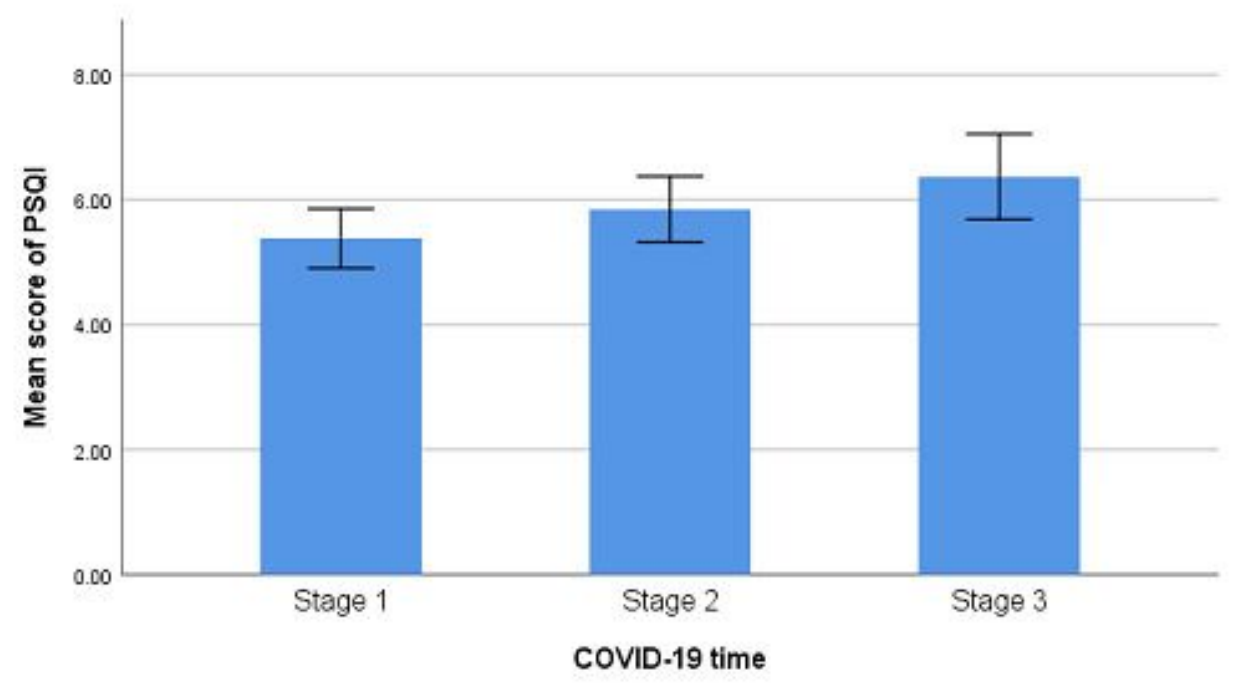

b

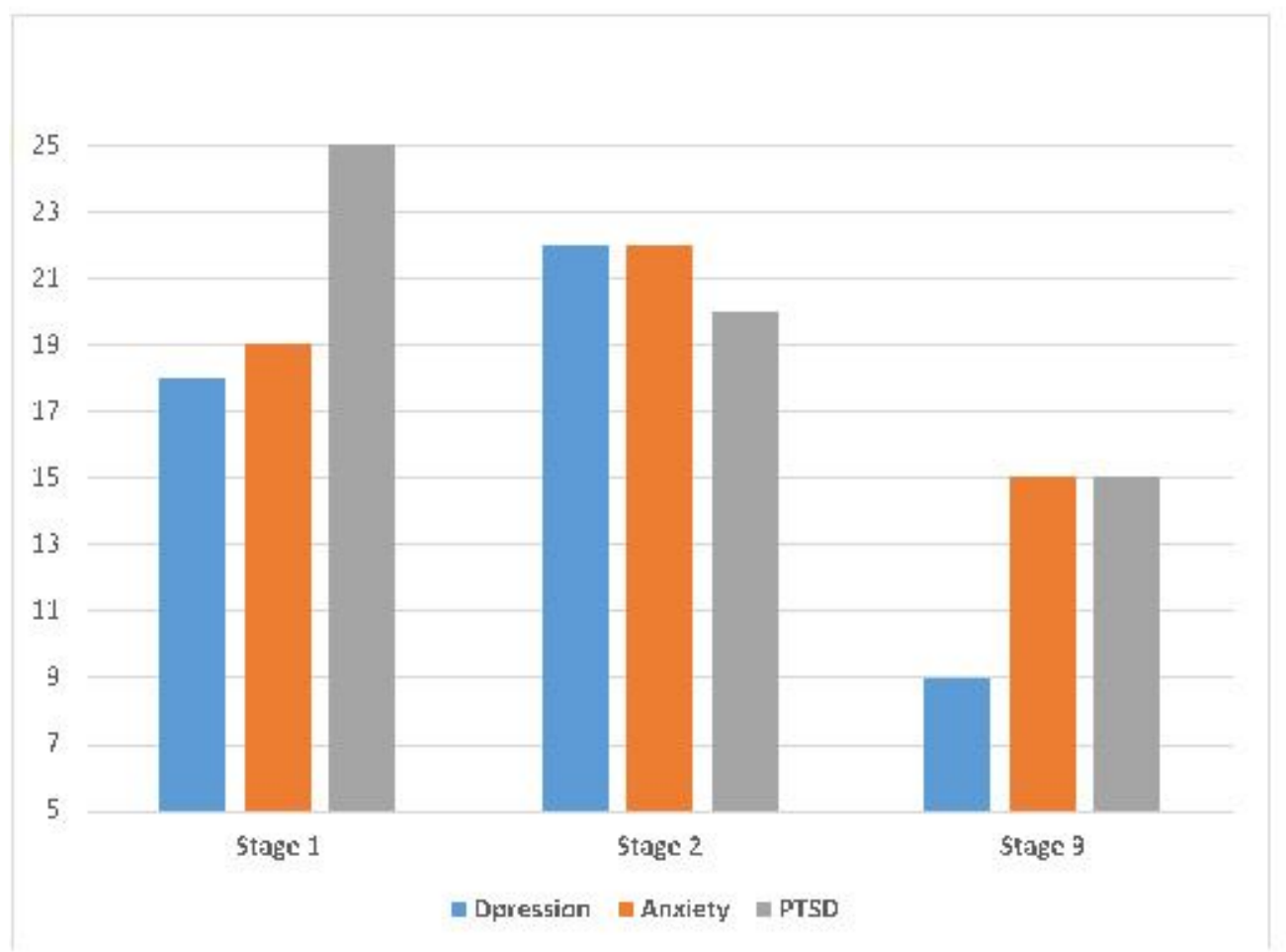

Figure 1

Sleep quality and mental disturbances at different COVID-19 epidemic stage. a. PSQI scores of HCWsat different stage $(p=0.042) ; b$. Number of HCWs with depression, anxiety and PTSD symptoms over time.

\section{Supplementary Files}

This is a list of supplementary files associated with this preprint. Click to download. 
- SupplementaryTable1.docx 